\title{
Application of wireless power system automation for electrical distribution system of Van, Turkey
}

\author{
Sabir Rüstemli a , Abdulkadir Özer b , Mehmet Nuri Almalı c , Mustafa Akdağ a* \\ a Bitlis Eren University, Electrical and Electronics Engineering Department, TR-13000, Bitlis Turkey \\ b Batman Provincial Administrations, Batman, Turkey \\ c Yuzuncu Yll University, Electrical and Electronics Engineering Department, Van, Turkey
}

\section{A R T I C LE INF O}

\section{Article history:}

Received 22 September 2017

Received in revised form 02 December 2017

Accepted 06 December 2017

\section{Keywords:}

Electrical power distribution system,

GSM based communication,

Power system automation Relays

Supervisory control

\begin{abstract}
A B S T RAC T
Reliable real-time information has become an important factor for safe delivery of power to the endusers and productivity of the electric utility and customer pleasure. Communication networks constitute the core of electrical system control applications in industry. The commercial and operational demands of electric utilities need a high-performance data communication network that improves the electrical system performance and provides possibility of a detailed study to predict failures. Supervisory Control and Data Acquisition (SCADA) systems are commonly used in industry for automation, control and data acquisition. Operators working in various units in an electric power company require access to SCADA data. Therefore, a design of cost-effective and reliable network architecture is a very important issue. Each network component has a unique address and the maximum quantity of components is restricted for the communication channel and protocol. In this paper, a power control application is studied by using wireless technology, and a SCADA system along with the MODBUS protocol which is most common communication protocol used in industrial automation systems. The application is carried out in a power distribution center found at the electrical distribution system located in Van, Turkey.
\end{abstract}

\section{Introduction}

As the term was introduced over 50 years ago first (Ebata et al., 2000), SCADA systems developed rapidly and widely used for monitoring and controlling of industrial applications from remote areas (Vigu et al., 2010). A SCADA software runs in a computer and it is necessary to supply data to software for controlling any equipment. (Lakhoua, 2010). As the distance of electrical generation and distribution centers and also customers spread over a wide area in an interconnected system, it becomes more difficult to transfer data to a SCADA center by wires or cables. In this case a new solution, as it is mentioned and presented in this paper, the GSM based wireless communication system is used to transfer data to SCADA centers and also to control distribution system components (Gagliarducci et al., 2007).

\footnotetext{
* Corresponding author. 
The main function of electric power system is to supply the system load requirements as economically as possible and with a reasonable degree of reliability and quality. The quality and availability required from electrical power is directly linked the power system reliability concept (Tanyi and Mbinkar, 2011). In order to improve the quality of the power with reasonable solutions, it is necessary to be acquainted with what sort of constraint has followed. Furthermore, once there is any insufficiency in the protection, control and monitoring of a power system, the system could become unstable. Thus, it requires a monitoring system that is able to automatically detect, monitor, typify and categorize the existing constraints on electrical lines. This provides some advantages to both end-users and utility companies (Daponte et al., 2004). Equipment failures, accidents, natural catastrophes and lightning strikes cause power disturbances and outages and frequently result in long service interruptions (Gungor et al., 2006; Mohagheghi et al., 2011). In the industry, having access to reliable data for the electrical power consumption of the facility is very important. The data of the previous and current performance of the main network variables can be employed for an on time diagnostic with respect to the possible irregularities in the facility. In addition to the data of the various electrical variables, it is more likely to increase the electrical system performance or do a comprehensive work to forecast potential failures (Conroy, 2001; Ramirez Leyva et al., 2004).

Analog circuits are characterized by circuit elements embedded inaccessibly within circuit chips. This is why a significant effort has been directed to develop fault diagnosis techniques which no longer need direct access to the circuit components of analog circuits. In recent years, developments in communication technologies have enabled reliable remote control systems that have the ability of monitoring the real time operating situations and performance of electrical systems (Gungor et al., 2006). The communication technology has its own advantages and drawbacks that must be evaluated to decide the best communication technology for electrical system control. To avoid potential disruptions in electrical systems because of unpredicted failures; a very robust, scalable, reliable and economical communication network between substations and a remote control center is vital (Ericsson, 2002; Zecevic, 1998).

The high performance communication network should also guarantee very strict quality of service necessities to avoid potential power disturbances and outages (Gungor et al., 2006; McGranaghan and Goodman, 2005). Industrial automation systems mostly use MODBUS protocol. MODBUS has been developed by MODICON Corporation and became a standard communication protocol (Ramirez Leyva et al., 2004).

Real-time monitoring and operational reliability in power distribution systems are very important (Lahti et al., 2011). SCADA systems are used to monitor, control and serve as a data storage of power system states. The functional range of systems like this incorporates data acquisition and basic data processing; process visualization through man machine interface as well as supplementary functions (secondary applications) depending on the concrete application purpose: control is achieved by SCADA command outputs (dispatcher actions, local processing, or secondary applications). Data logs are used for analyzing and testing the facility. SCADA systems are to provide solutions for real-time environment and designed as distributed systems (Ericsson, 2004; Sagi et al., 2012). Functional computers are linked via local area network, usually with Ethernet. These computers are redundantly organized to meet high reliability demands (Chainey and Block, 1994). Many companies consider SCADA systems to access real time data monitor and also alarm, trend, and report functions from remote terminal by using various communication media such as Internet, Private Leased Line (PLL), Dial-up network, satellite etc. Latest technological developments have made location transparency reachable through the Internet at a reasonably low cost and suitable level of security. Some of the possibly esteemed progresses are Intranet and Extranet. Currently, developments in Graphical User Interface (GUI) of the SCADA have justly high resolution graphical animations (Karacor and Ozdemir, 2004).

Generally, a SCADA system comprises of a set of various master units (MU) and Remote Terminal Units (RTU) (Wu et al., 2005). Each RTU is installed in some points and gathers accurate data. The MU requests data to control each RTU, saves the data and the MU can perform a precise action to control a section of the facility. Entire data is sent through a communication channel, frequently through a shared low speed wireless channel (Chainey and Block, 1994).

The substations are controlled from the control center when the SCADA system is used. Thus, maintenance time and labor decrease. General structure of SCADA system is presented in Appendix.

MU, RTU and communication systems are determined to design SCADA system. In this study; GSM, Secondary relay with microprocessor, Server PC, Modem and MODBUS protocol found in the breaker measurement cabin are used to carry out the control process. The communication is established via RS 485 serial port. The aim of the study is to provide the power control by using wireless technology. Wireless technologies offer not only significant benefits over wired; they are also more suitable for remote end applications due to low installation cost, mobility, rapid deployment (Parikh, 2010). The work is prepared as follows: In section 2, equipment is presented. In Section 3, the method is discussed. In section 4, the SCADA Application is described. In section 5, discussion and evaluation are presented. Lastly, the work is concluded in section 6 .

\section{Equipment}

The main set of equipment of this study consists of overhead transmission line (OTL) breakers, OTL current transformers, OTL voltage transformers, OTL control panel, modem, RS485, computer and electronic relays which are suitable for wireless 
communication. The characteristics of these components are presented as follows:

\subsection{Relay}

In this application, MC-30R type relay is used. It is controlled using the four keys on the LCD, RS-232 port or RS-485 port. The relay is secondary relay with microprocessor. Analog signals in the input of the relay are converted into digital signals and transmitted to microprocessor. Open-Close commands are sent to the breakers by the relays from remote and nearby distance via the communication port.

Relays communicate by using IEC 6080-103 protocol over RS485 port. There is a RS-232 port in front of the relay. The relay has a memory. Adjustment of the relay is stored in the memory. It can be selected according to IEC and ANSI standard. There are constant time and inverse time characteristics of the relay. Open-Close command can be sent to the breakers by the relays from remote and nearby distance via the communication port. It can be programmed re-closing characteristic for rural area if it is necessary.

\subsection{Breakers}

A SF6 breaker $(15 \mathrm{kV}, 630 \mathrm{~A}, 12.5 \mathrm{kA})$ is used in this study. There is SF6 gas in the electrical arc putting out region. This breaker is reliable. The gas can be used for a long time. Dielectric coefficient is high. Mechanical structure is wellbuilt. It is cheap. Open-Close number is high. It is widely used in Turkey. The pressure of the SF6 breaker is 2 or 3 bar. The breaker maintains low pressure while the arc is occurred. There is no explosion or gas burn.

\subsection{Measurement Transformer}

Two measurement transformers are used. The current transformer is dry-type and 100/5-5 A. Voltage transformer is dry-type, $15 / 0.1 \mathrm{kV}$ and has a power of $800 \mathrm{VA}$.

\subsection{Computer}

Computer is a unit that provides control and coordination of additional units. In this application, a basic laptop computer is used.

\subsection{Modem}

Siemens MC35i Terminal type GSM modem is used in the application.

\subsection{Uninterrupted Power Supply}

AC/DC power supplies should be included in the control center to power up necessary equipment. Its power predefined of $1500 \mathrm{VA}, \mathrm{AC} / \mathrm{DC}$ single phase input and two phase output.

\section{Method}

SCADA application is carried out by means of GSM. Applied control is executed by using secondary relay interface program. The relay uses MODBUS remote communication protocol. The performed application can be detailed with the stages mentioned below:

1. The principle schema of the SCADA is figured out via the single-line schema of distribution network (Figure 2).

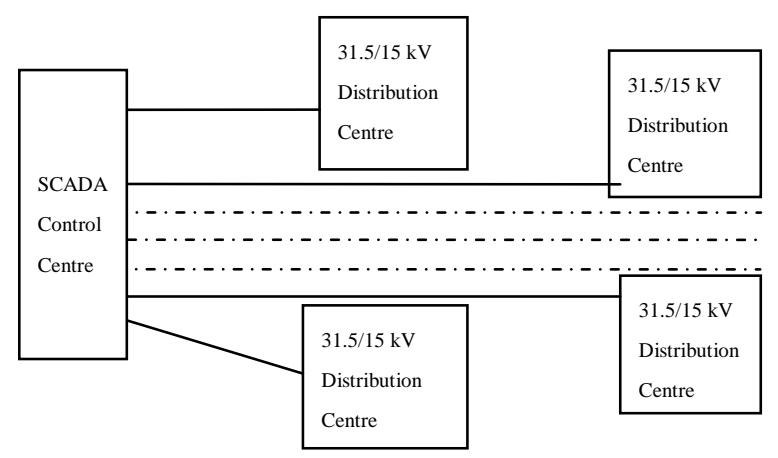

Figure 2. The determined SCADA principle schema of OTL

2. GSM modems, RS485/232 converter, single computer, printer, un-interrupt power supply, two GSM line, the secondary relay and interface program are prepared for the control system.

3. A block diagram, which shows the structure of the SCADA system, is given in Appendix.

4. Firstly, interface program of the secondary relay is prepared for SCADA application. The relay is used as RTU. The relay interface program is used as RTU software. The relay uses MODBUS remote communication protocol in which system security by using a secret code.

5. GSM modem is registered on the computer. It is necessary for the computer to recognize the GSM modem.

6. The secondary relays are connected to the all outputs found in the distribution centre.

7. Connections of the secondary relays are carried out by using RS485/232 converter over serial port.

8. UPS connecting is made for computer of the main control unit using DC power supply in the distribution centre.

9. The system and function adjustments of the relays are made by determining current transformers rates in the outputs of the distribution centre. The relay coordination is made with the adjustments of the relay found in inputs and outputs of the distribution centre. Thus, the faults occurred in outputs do not interrupt the power of the whole distribution centre. 
10. The connection schema of the SCADA application is formed. This schema is given in Figure 4.

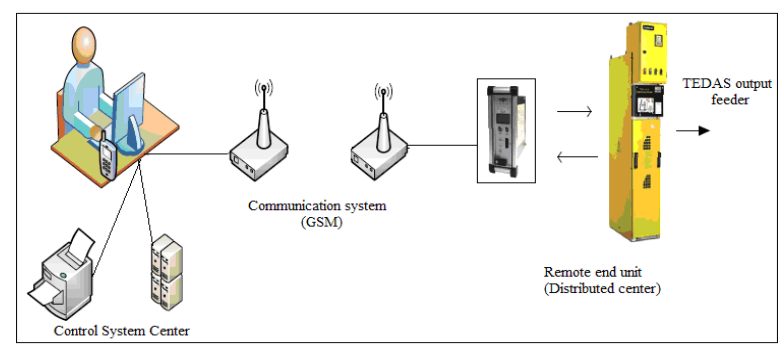

\section{SCADA Application}

SCADA application can be divided into three parts: carrying out the control, acquire data from remote area and controlling the system. There are file, search, relay options, tools, language, window and help menus in the main page of the control program of the SCADA application. The main page picture is shown in Figure 5.

Figure 4. The connection schema of the SCADA application

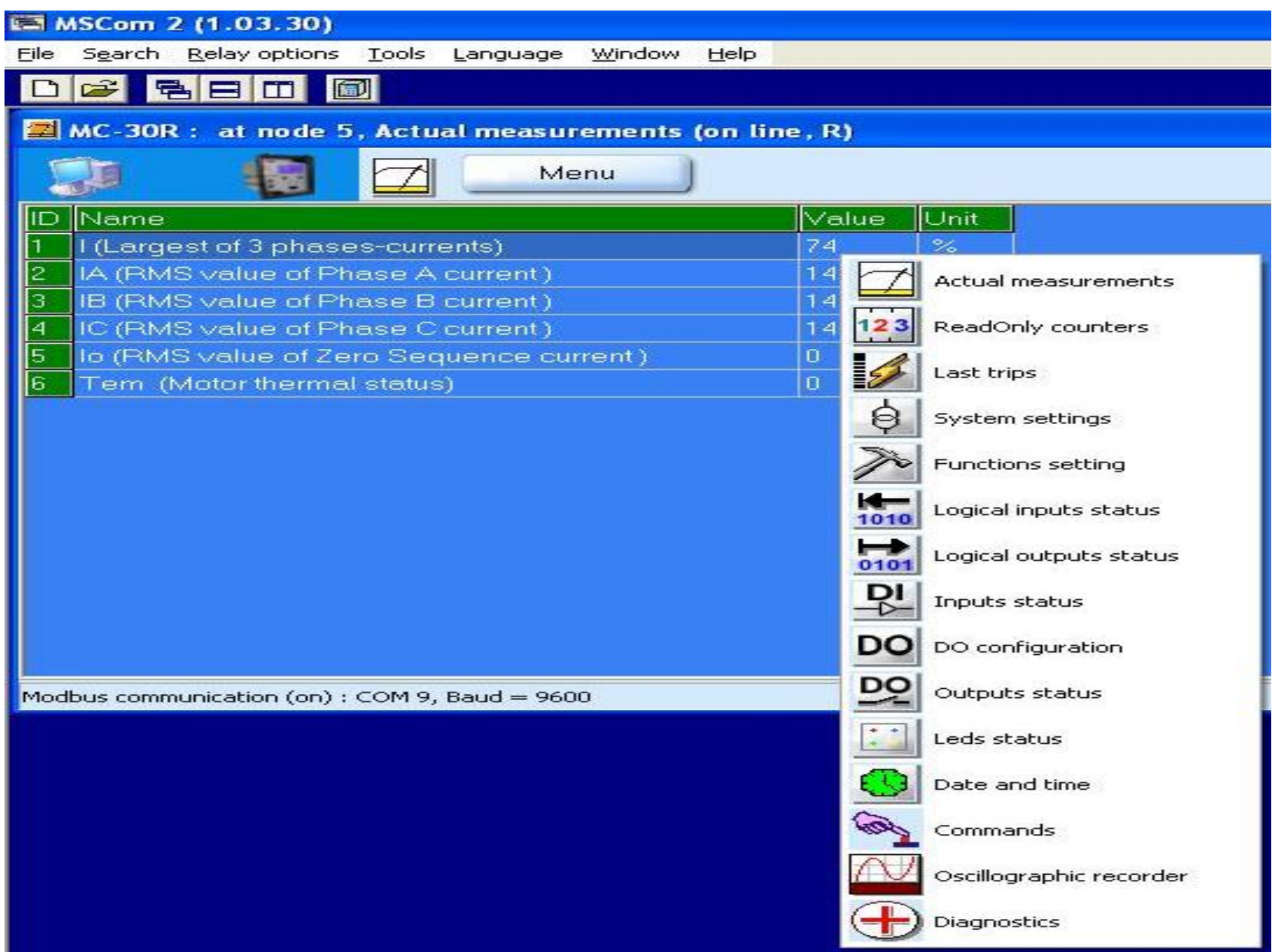

Figure 5. Main Menu

Selection of language, relay model, setting up of the modem, passing the other menus and closing the system can be made by using the main page.

There are new, open and exit selections in file menu to select the relay model, to load relay adjustments which is saved in the computer the program and to exit from the program, respectively. There are call menu, relay selection menu and window menu for adjustments of relay setting up in serial port, to array the relays of the outputs in the distribution center by address number and to watch the status of the relays which are connected as symbolic. 


\subsection{Setting up the Modem}

The processes below are made to communicate with GSM modem found in Distribution Center 5 (DC-5) using MC-30R interface program of the secondary relay found in the communication center.

1. Call over serial port.

2. Determine communication rate of the modem (19200 MHz).

3. Long (GSM $200 \mathrm{~ms}$ ) is determined as time-out for setting up of the communication by the modem.

4. The command "setup" is given for connecting to the modem. The connection with relay found in the distribution center is given Figure 6.

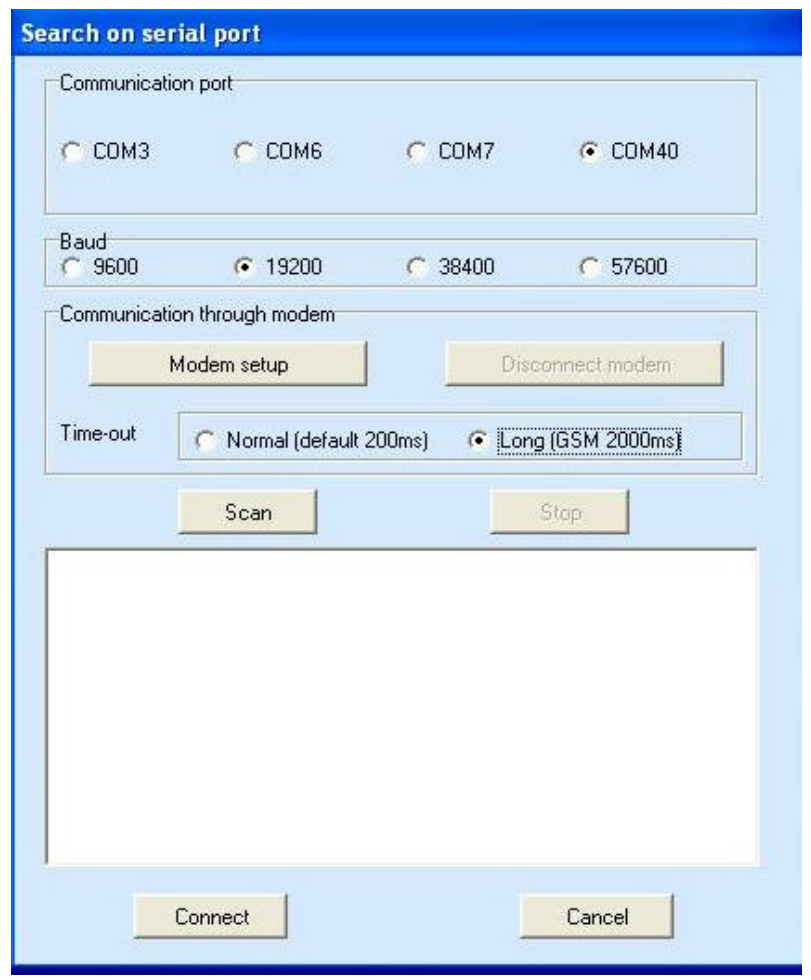

Figure 6. Connection with relay

5. Phone number of the GSM modem found in the distribution center is entered.

6. The connection is set up with the modem.

7. The connection is provided by modem found in DC5.

8. The defined relays in the outputs of the distribution center are shown on the screen according to address row by means of call command in network. There are 11 overhead transmission line outputs of the SCADA system. System operators can interfere in the system from the remote distance by using interface.
The defined relays in the outputs of the distribution center are shown on the screen according to address row by means of call command in network. There are 11 OTL outputs of the SCADA system. System operators can interfere in the system from the remote distance by using interface.

The electrical distribution network in the study is supplied by $154 / 31.5 \mathrm{kV}$, from Van TM. The Output feeders of the OTL are supplied from Van TM. One of the feeders supplies Distribution Centre 2 (DC-2). DC-5 is supplied by OTL network of DC-2.

The application is made in DC-5. The below outputs are supplied from DC-5 (Table 1).

Table 1. DC-5 Outputs

\begin{tabular}{lllll}
\hline Address & Relay & Transformer & $\begin{array}{l}\text { Voltage } \\
\mathbf{( k V )}\end{array}$ & Address \\
\hline 1 & MC30-R & TR-1 & 30 & 1 \\
2 & MC30-R & TR-2 & 30 & 2 \\
3 & MC30-R & insulating & 30 & 3 \\
& & switch & & \\
4 & MC30-R & TR-16-45 & 15 & 4 \\
\hline 5 & MC30-R & TR-99 & 15 & 5 \\
\hline 6 & MC30-R & TR-98 & 15 & 6 \\
\hline 7 & MC30-R & TR-114 & 15 & 7 \\
\hline 8 & MC30-R & TR-106 & 15 & 8 \\
\hline 9 & MC30-R & TR-YEM & 15 & 9 \\
\hline
\end{tabular}

The outputs connected to the modem according to the address are shown in Figure 7.

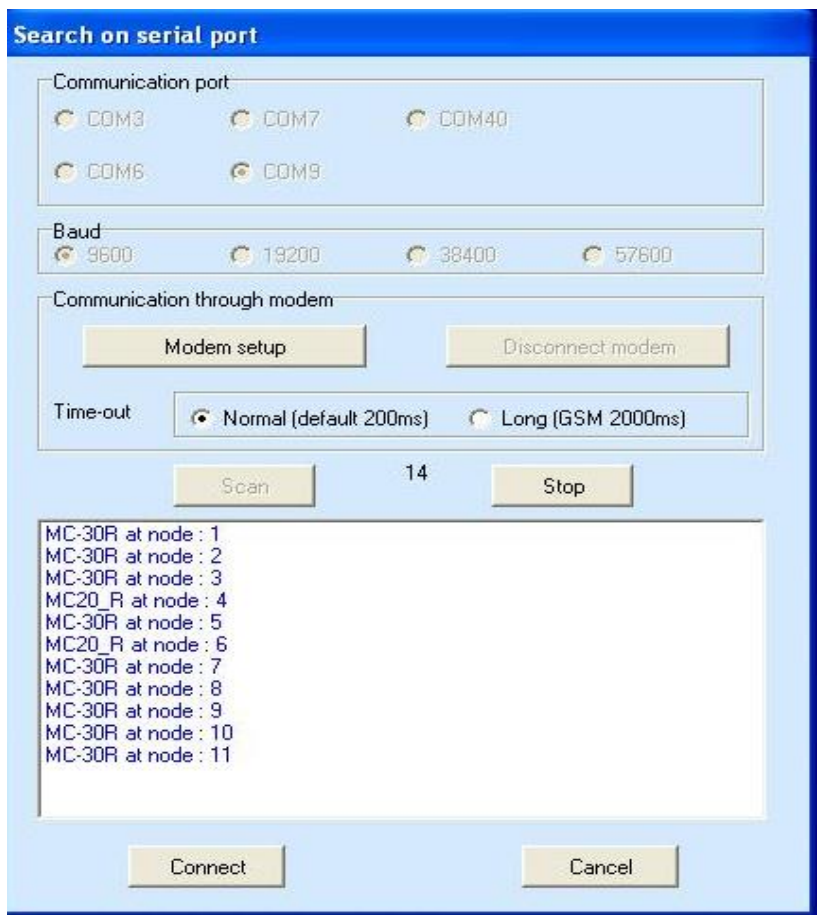

Figure 7. The outputs according to address number 


\subsection{Selection of the Relay to be controlled}

Choosing the relays to be remote control, it is passed interfere in mod by connecting command. Every relays can be observed on the screen at the same time by connecting the relay one by one. Selection of the relay to be connected is demonstrated in Figure 8 .

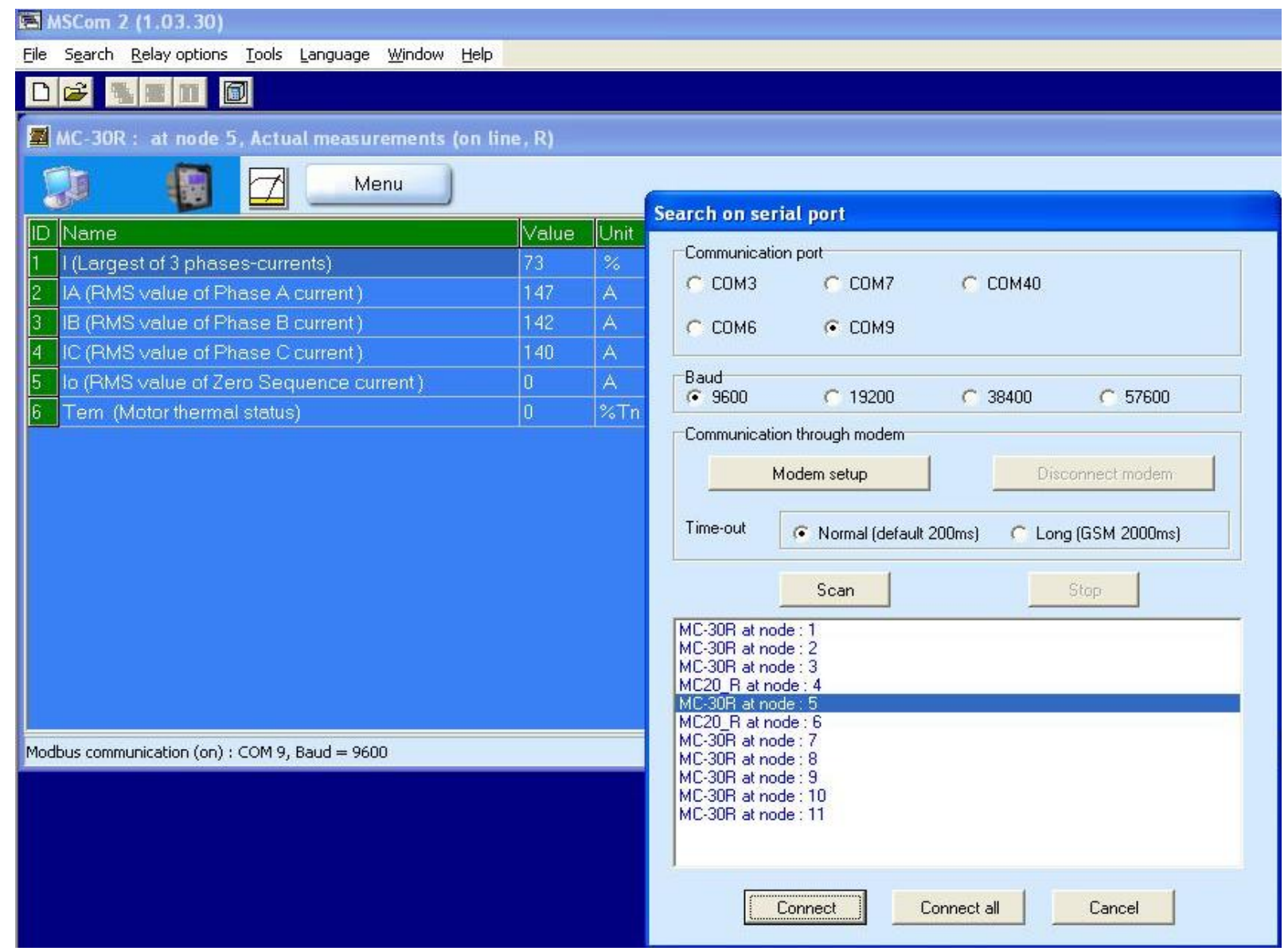

Figure 8. Selection of the relay to be controlled

\subsection{Menu}

The main menu provides passing the other menus. Needed process for changing or observing of adjustments of the relay to be remote control is made by means of the main menu. This menu is shown in Figure 5.

\subsection{Instantaneous measurements}

The main menu is clarified as follows:

1. It shows the loading rate of the feeder according to selected current transformer as percent.

2. It shows the primer RMS value of phase A current.

3. It shows the primer RMS value of phase B current.
4. It shows the primer RMS value of phase $\mathrm{C}$ current.

5. It shows the current I0 which flows through the neutral wire in the wye (Y) point. Each of the windings of three-phase transformer is joined at wye point. The relay sends the on command from the ground protection to the breaker when the current go through over the tuning value. The tuning value is maximum $20 \%$ of the current through the phases.

6. TN shows the rate of heat increment under full load as percent. Instantaneous values of the selected relay are given in Figure 8. 


\subsection{Sending command to the breaker}

The warning message, "is the command sending to the relay", appears on the screen if the command on send the breaker whereas the breaker position is off. If yes is selected the process will be carried out. The process of sending of the command to the relay is pictured in Figure 9.

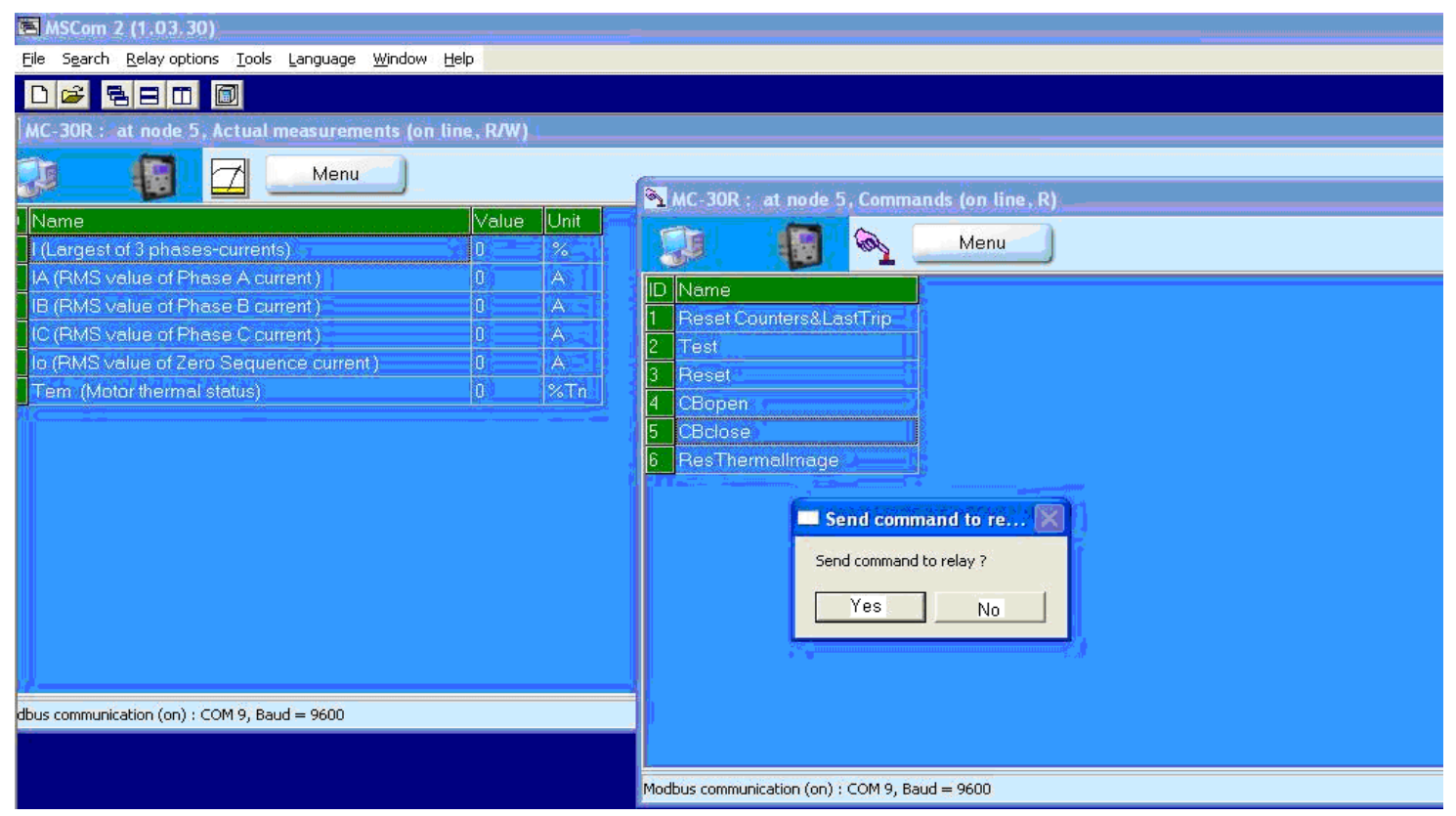

Figure 9. Sending command to the relay

\section{Discussion and Evaluation}

It is possible to analyze the behavior of all the electrical power consumption by SCADA. GSM based SCADA control provides an economic solution to control and automate the information flow for small to medium size power distribution systems. It can be made by the proposed mobile phone based SCADA system. In general, properties of the system can be stated as:

- State information of the relay can be controlled.

- $\quad$ The currents belonging to every phases and state of rate of the feeders according to state of rate of the loading can be observed by instantaneous measurement menu.

- Position of the breaker, A/A ground (open) signal and A/A phase (open) signal according to fault type, and state of supplying of the relay can be observed by operator.

- It can be tested whether the signals on the relay are active or not.

- The signal given by relay according to fault type can be eliminated.
- Open-Close commands can be sent to the breaker after confirming the secret code.

- Tuning of the over current protection, tuning of the ground leakage and determining of the current-time curve can be made.

- $\quad$ Re-closed tuning and process can be made.

- Communication parameters can be adjusted.

- The serial number of the relay can be controlled.

- The user secret code can be given to send the command (Open-Close) or to change the relay adjustment.

- The rate of the current transformers and frequency can be observed by operator.

- Continuous current of the feeder can be observed.

- The date and time information can be followed.

- What kind of the fault is occurred and how many reclosed related to the same fault type is occurred can be observed. 
- $\quad$ The date, time, fault type, IA, IB, IC and I0 belonging to last twenty opening can be watched.

\section{Conclusions}

The core of the electrical system control applications is the communication network. Therefore, the design of costeffective and reliable network architecture is very important and vital. The most effective way of carrying out of this goal is to set up the control system in the whole electrical network. SCADA systems provide centralized control of systems from a single command center. Especially in power distribution systems SCADA can provide remote control and command of a system that covers a wide geographical area. By monitoring the power systems remotely by SCADA can provide quick respond to power outages. So effects of unplanned power outages on customers and also on generation units can be minimized.

In this paper, an application is designed to control and manage the system from a single center by using SCADA system. The aim of application is to decrease the power outage time to minimum by using SCADA system. A relay and breaker control based SCADA application for immediate re- activation is designed to reach this aim. First, the materials which controlled by software is introduced. Then block diagram of entire system is formed. Modem based design of SCADA allows the GSM controlling option. After the necessary settings of both hardware and software, GSM controlled SCADA system is performed for the electrical distribution system in Van/Turkey. GSM controlled SCADA system gave appropriate results on the distribution system.

Interconnected network components, which are spread over a wide area, can be controlled wirelessly by using the proposed system. This allows a quick interfere to possible service interruptions making outage periods decrease. Power outage period may last for hours when classical control systems are used. These interruptions have a lot of adverse effects on customers. Power outage time improved to a few minutes for some cases after system has been used. As the control is performed in a single center, necessary number of technical staff is also decreased.

\section{Appendix}

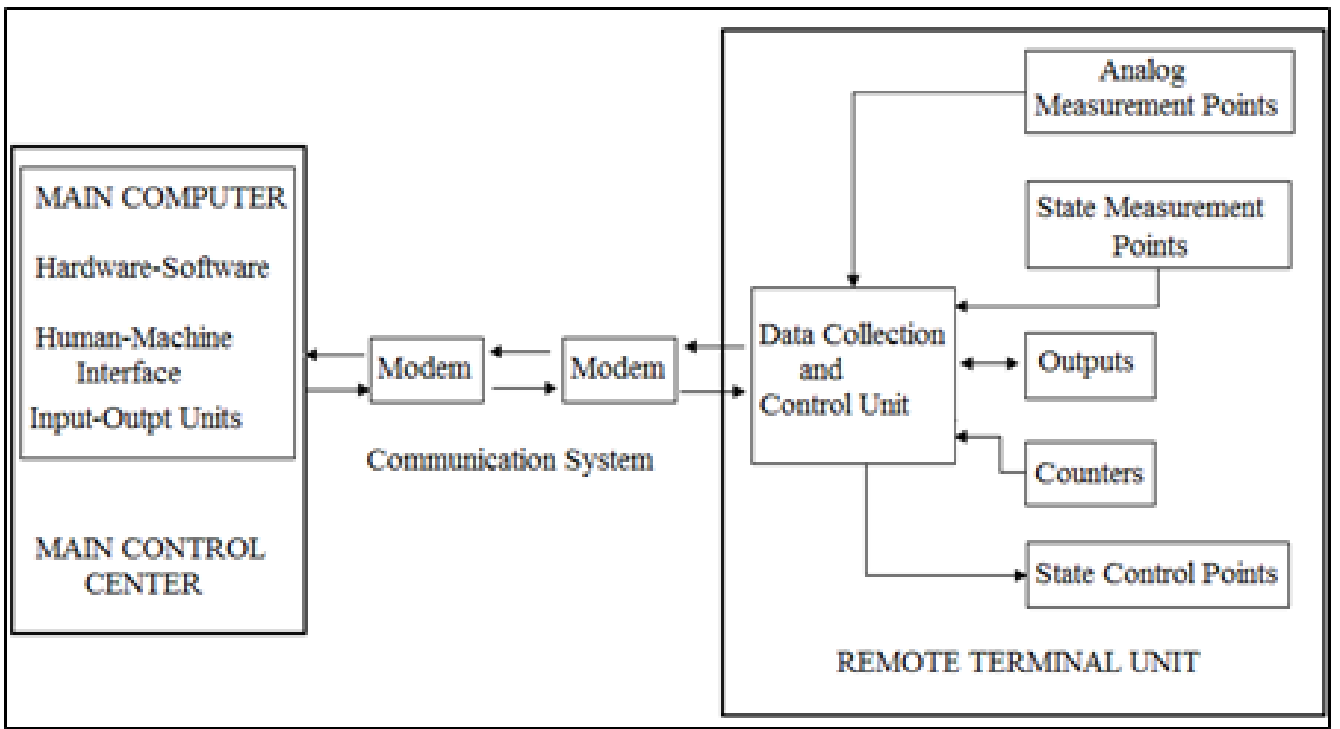

General structure of SCADA system 


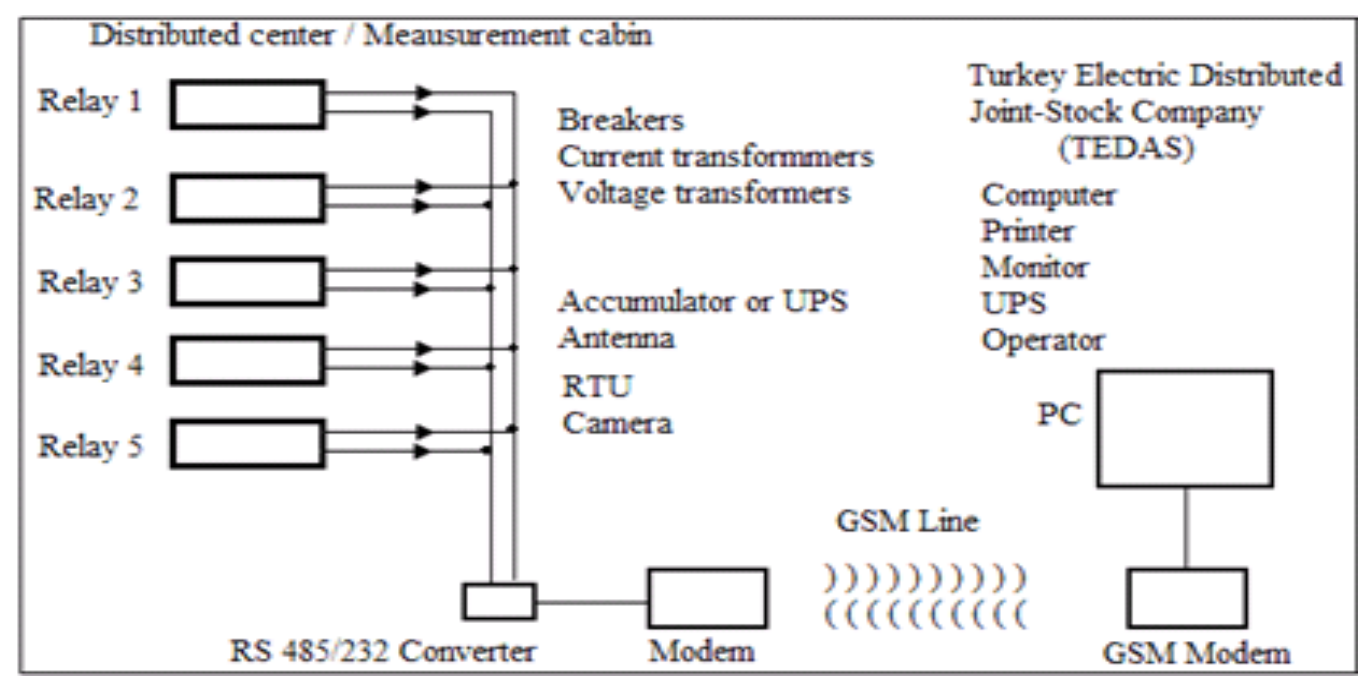

Block diagram of the SCADA system

\section{References}

Chainey, W.E., and Block, W.R., 1994. Recent advances in master station architecture. IEEE Computer Applications in Power, 7(2), 2429.

Conroy, E., 2001. Power monitoring and harmonic problems in the modern building. Power Engineering Journal, 15(2), 101-107.

Daponte, P., DiPenta, M., and Mercurio, G., 2004. TransientMeter: A Distributed Measurement System for Power Quality Monitoring. IEEE Transactions on Power Delivery, 19(2), 456-463.

Ebata, Y., Hayashi, H., Hasegawa, Y., Komatsu, S., and Suzuki, K., 2000. Development of the Intranet-based SCADA (supervisory control and data acquisition system) for power system. IEEE Power Engineering Society Winter Meeting 2000, Singapore-Singapore, 23-27 January, p. 1656.

Ericsson, G.N., 2002. Classification of power systems communications needs and requirements: experiences from case studies at Swedish National Grid. IEEE Transactions on Power Delivery, 17(2), 345-347.

Ericsson, G.N., 2004. Communication requirements-basis for investment in a utility wide-area network. IEEE Transactions on Power Delivery, 19(1), 92-95.

Gagliarducci, M., Lampasi, D.A., and Podestà, L., 2007. GSM-based monitoring and control of photovoltaic power generation. Measurement, 40(3), 314-321.

Gungor, V.C., and Lambert, F.C., 2006. A survey on communication networks for electric system automation. Computer Networks, 50(7), 877-897.

Karacor, M., and Ozdemir, E., 2004. Mobile Phone-Based SCADA Automation. Measurement and Control, 37(9), 268-272.

Lahti, J.P., Shamsuzzoha, A., and Kankaanpaa, T., 2011. Web-based technologies in power plant automation and SCADA systems: A review and evaluation. Int. Conf. on Control System, Computing and Engineering 2011, Penang-Malaysia, 25-27 November, p. 279.
Lakhoua, M.N., 2010. Surveillance of pumps vibrations using a supervisory control and data acquisition system. Journal of Control Engineering and Applied Informatics, 12(1), 15-20.

McGranaghan, M., and Goodman, F., 2005. Technical and System Requirements for Advanced Distribution Automation. 18 $8^{\text {th }}$ Int. Conf. and Exhibition on Electricity Distribution (CIRED 2005), Turin-Italy, 6-9 June, v 5, p. 93.

Mohagheghi, S., Tournier, J-C., Stoupis J., Guise, L., Coste, T., Andersen C. A., and Dall J., 2011. Applications of IEC 61850 in distribution automation. Power Systems Conference and Exposition (PSCE), 2011 IEEE/PES, Phoenix, AZ, USA, 20-23 March, p. 1.

Parikh, P.P., Kanabar, M.G., and Sidhu, T.S., 2010. Opportunities and challenges of wireless communication technologies for smart grid applications. IEEE Power and Energy Society General Meeting, Providence, RI-USA, 25-29 July, p. 1.

Ramirez Leyva, F.H., Cuellar, J.P.A., Basilio, R.G.M., and Justo, E.E., 2004. Wireless system for electrical networks testing based on MODBUS protocol. 14th Int. Conf. on Electronics, Communications and Computers, Veracruz, Mexico-Mexico, 16-18 February, p. 58.

Sagi, M., Atlagic, B., Milinkov, D., Bogovac, B., and Culaja, S., 2012. High-performance distributed SCADA system architecture. 16 $6^{\text {th }}$ Mediterranean Electrotechnical Conference, Yasmine HammametTunisia, 25-28 March, p. 152.

Tanyi, E., and Mbinkar, E., 2011. A Wide Area Network for Data Acquisition and Real-time Control of the Cameroon Power System. Journal of Control Engineering and Applied Informatics, 13(1), 5-11.

Vigu, C., Gota D.I., and Capatana D., 2010. Improving the wind speed estimation algorithms using results obtained in the field. Journal of Control Engineering and Applied Informatics. 12(3), 13-17.

Wu, F.F., Moslehi, K., and Bose, A., 2005. Power System Control Centers: Past, Present, and Future. Proc. of the IEEE, 93(11), 1890.

Zecevic, G., 1998. Web based interface to SCADA system. Int. Conf. on Power System Technology, Beijing China, 18-21 August, p. 1218. 